\title{
Redescripción de Dichotomius camposeabrai y D. nemoricola (Coleoptera: Scarabaeidae: Scarabaeinae), con apuntes sobre su posición sistemática
}

\section{Redescription of Dichotomius camposeabrai and D. nemoricola (Coleoptera: Scarabaeidae: Scarabaeinae), with notes on their systematic position}

\author{
Jorge Armando Arias-Buriticá ${ }^{1 \otimes}$ y Fernando Z. Vaz-de-Mello² \\ ${ }^{1}$ Instituto de Ciencias Naturales, Universidad Nacional de Colombia. Entrada Calle 53, $2^{\circ}$ edificio, Apartado 7495, Bogotá, Colombia. \\ ${ }^{2}$ Departamento de Biologia e Zoologia, Instituto de Biociencias, Universidade Federal de Mato Grosso. Av. Fernando Correa da Costa, 2367 Boa \\ Esperança, Cuiabá MT 78060-900, Brasil. \\ \joaariasbu@unal.edu.co
}

\begin{abstract}
Resumen. Se presentan redescripciones de Dichotomius camposeabrai Martínez, 1974 y D. nemoricola (Pereira, 1942) que incluyen la descripción de la hembra de la primera especie y del macho de la segunda, hasta ahora desconocidos. Para cada especie se realizaron ilustraciones del habitus del macho, del órgano genital masculino y de los escleritos del saco interno, así como de la cabeza y pronoto de las hembras. Se proporcionan datos sobre la distribución geográfica y algunos comentarios de las especies. Además, se propone y discute la transferencia de $D$. camposeabrai al grupo bitiensis, dadas las similitudes de la morfología externa y del órgano genital masculino que presenta con $D$. nemoricola.

Palabras clave: órgano genital masculino, escleritos del saco interno, lamela copulatriz, grupo bitiensis.

Abstract. The redescription of Dichotomius camposeabrai Martinez, 1974 and D. nemoricola (Pereira, 1942) is presented, including the description of the female of the first species and the male of the second so far unknown. For each species, illustrations of the male habitus, male genital organ, the sclerites of internal sac and head and pronotum of female are presented. Information about geographical distribution and some comments are available. In addition the transfer of $D$. camposeabrai to the bitiensis group is proposed and discussed, given the similarities of the external morphology and male genital organ with those of $D$. nemoricola.
\end{abstract}

Key words: male genital organ, sclerites of internal sac, copulatrix lamella, bitiensis group.

\section{Introducción}

Dichotomius Hope, 1838 es uno de los géneros más diversos y abundantes de escarabajos coprófagos (Coleoptera: Scarabaeidae: Scarabaeinae) en los ecosistemas neotropicales con alrededor de 154 especies distribuidas desde el noroeste de Estados Unidos de América hasta Argentina central. Luederwaldt (1929), basado en características de la morfología externa, propuso la división de las especies de Dichotomius en secciones (aquí tratadas como grupos) para abordar su estudio taxonómico. Sin embargo, en la actualidad se han encontrado inconsistencias y dificultades en la ubicación de algunas especies en los grupos propuestos por este autor; siendo Dichotomius uno de los géneros que necesita con mayor urgencia de una revisión taxonómica dentro de los Scarabaeinae (Génier, 2000; Vaz de Mello et al., 2001; Kohlmann, 2003; Gandini y Aguilar, 2009).

Recibido: 01 agosto 2011; octubre 2011
En los grupos del género, presentan inconsistencia en la ubicación Dichotomius camposeabrai Martínez, 1974 y Dichotomius nemoricola (Pereira, 1942), especies del subgénero nominotípico, propuestas después de Luederwaldt (1929) y ubicadas en 2 distintos grupos de especies, la primera en buqueti y la segunda en bitiensis. Son especies raras en colecciones, las cuales sólo se conocían de la descripción original, donde no fueron descritos la hembra de $D$. camposeabrai y el macho de $D$. nemoricola; a esto se suma el desconocimiento de los hábitos ecológicos y distribuciones de estas especies (Martínez, 1974; Pereira, 1942).

En este trabajo se redescriben Dichotomius camposeabrai Martínez, 1974 y D. nemoricola (Pereira, 1942) y se describen los sexos, hasta ahora desconocidos, de cada una de las especies. Además, con base en datos de la morfología externa y de los órganos genitales masculinos, se presentan evidencias de una relación estrecha entre estas 2 especies, por lo cual, se propone la transferencia de $D$. camposeabrai al grupo bitiensis del cual es parte $D$. nemoricola. 


\section{Materiales y métodos}

El estudio se basó en la revisión de 13 ejemplares depositados en las siguientes colecciones entomológicas (acrónimo y nombre del curador entre paréntesis): Seção de Entomologia da Coleção Zoológica da Universidade Federal de Mato Grosso, Cuiabá, Brasil (CEMT - Fernando Vaz-de-Mello); Canadian Museum of Nature, Ottawa, Canadá (CMNC - François Génier) y Museu de Zoologia da Universidade de São Paulo, São Paulo, Brasil (MZSP Sonia Casari y Carlos Campaner).

Los caracteres fueron revisados con un estereomicroscopio marca Leica $(100 \times)$. Para la preparación de los ejemplares, se siguió la metodología propuesta por Medina et al. (2003). Para la disección y preparación de los órganos genitales masculinos (aedeagus), se siguió la metodología propuesta por Zunino (1978). Se realizaron ilustraciones del habitus del macho y de las diferencias presentes en la cabeza y pronoto de las hembras, así como el esquema del aedeagus en vista lateral, dorsal, ventral y de los escleritos del saco interno. La terminología para la morfología externa e interna se trabajó de acuerdo con Edmonds (1972) y la de los órganos genitales masculinos con la de Zunino (1978). Con los datos de las etiquetas de localidad de los ejemplares se elaboró el mapa de distribución, utilizando el programa DIVA GIS ver. 5.2 (Hijmans et al. 2004).

\section{Redescripciones}

Dichotomius camposeabrai Martínez, 1974 (Fig. 1)

Holotipo. Probablemente en el Museo Argentino de Ciencias Naturales Bernardino Rivadavia, Buenos Aires. No examinado, por disponerse de ejemplares procedentes de un área geográfica cercana a la localidad tipo, que fueron comparados con este holotipo por el segundo autor Localidad tipo. Brasil: Espírito Santo: Linhares, Parque Nacional Sooretama.

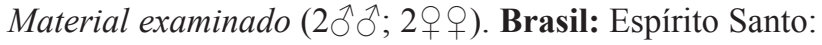
Linhares, X/2004, Grossi, P. (1 ${ }^{\top}$; 1 우) [CEMT]; Minas Gerais: Caratinga, dic[iembre], [1]980, Coll: Martínez.

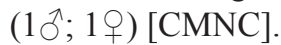

Machos. Con cuerpo de 15.0-15.5 mm de longitud y 8.8$8.9 \mathrm{~mm}$ de anchura (Fig. 1A). Coloración negra, con sedas castañas en los bordes laterales de la cabeza y pronoto.

Cabeza. Vez y media más ancha que larga, con sedas más largas en la parte posterior y de menor tamaño en la anterior. Margen anterior ovalado y truncado, con un reborde en toda la extensión del clípeo, en el centro 2 dientes clipeales suaves. Superficie del clípeo en toda su extensión con callosidades suaves dando la apariencia de corrugado y convirtiéndose en puntos suaves cerca del proceso cefálico. Sutura clípeo-genal evidente, llegando hasta el proceso cefálico. Genas con borde lateral recto y luego curvo, superficie similar a la del clípeo. Frente con un proceso cefálico transversal situado entre los ojos, de forma triangular, ancho en su base y terminando en un proceso coniforme de punta roma; parte posterior de la frente con hoyuelos alargados y profundos separados entre sí por 1 vez su anchura.

Tórax. Pronoto 2 veces más ancho que largo, con sedas evidentes en los bordes laterales. Superficie con puntuaciones pequeñas y separadas entre sí por al menos 2 a 3 veces su diámetro, redondas en la parte posterior y alargadas hacia las zonas laterales. Parte anterior con un borde delgado y liso, que se comprime hacia los ángulos anteriores, los cuales son salientes y redondeados. Borde antero-lateral recto formando un ángulo de $120^{\circ}$ con el borde lateral posterior. En la parte central presenta una excavación que va desde la parte anterior hasta los $2 / 3$ del mismo, formando una protuberancia a cada lado. Fóveas laterales alargadas y profundas, debajo de las mismas se forma un pliegue en la parte lateral. Hipómero con superficie chagrinada y puntos ocelados grandes separados por una distancia de menos de 1 vez su diámetro; de algunos sale 1 seda erecta, larga y castaña; en la parte central debajo del fémur la superficie es brillante, parte lateral con hileras de sedas largas que sobresalen siendo evidentes en vista dorsal. Prosterno chagrinado con puntos setígeros dispersos en los bordes laterales. Coxas de las patas anteriores con superficie muy chagrinada; fémur en vista ventral brillante, liso y con puntuaciones pequeñas, separadas entre sí por al menos 3 veces su diámetro, zona lateral cercana a la inserción de la tibia con 4 a 6 puntos setígeros fuertes, de cada uno sale 1 seda erecta y larga de color castaño; borde posterior con muchas sedas largas y castañas; desde la inserción del trocantín salen 2 quillas paralelas al fémur, una llega a la parte apical y la otra al surco del fémur con muchas sedas que se vuelven más densas hacia el surco; tibia con 4 dientes laterales y superficie chagrinada, parte media más elevada, de allí salen 3 quillas hacia los dientes tibiales anteriores; en la parte central hay una hilera de puntos setígeros grandes y profundos, de cada uno sale 1 seda erecta y larga, luego continua como una quilla en el borde externo también con sedas, interrumpida en los dientes tibiales; espolón tibial en forma de L invertida, que va hasta el tercer tarsómero, aplanado ventralmente y convexo en la parte dorsal; con 5 tarsómeros, el primero y quinto subcuadrangulares, tan largos como el segundo y tercero unidos, los cuales son subovoides, con sedas en el borde apical; el quinto en el borde apical y ventral con sedas, terminando en 2 uñas simples. Élitros con la superficie de las interestrías chagrinada con puntos poco profundos y pequeños, separados entre sí por al menos 3 veces su diámetro; estrías bicare- 
nadas con puntos ocelados pequeños en toda su extensión, separados aproximadamente 3 veces su diámetro; húmero pronunciado en la parte anterior de las estrías 6 y 7; epipleura separada por una carena fuerte, de superficie chagrinada. Mesosterno reducido en su parte media, en la cual hay un proceso liso y brillante que no deja ver en su parte central la sutura meso-metaesternal, presenta hacia los lados una superficie chagrinada con puntos ocelados grandes, separados entre sí menos de 1 diámetro. Mesepisterno con la misma superficie que los bordes laterales del mesosterno, pero de cada punto ocelado sale 1 seda erecta y larga. Patas medias con coxas con superficie muy chagrinada; fémur medio en vista ventral con superficie brillante, lisa y con puntuaciones pequeñas separadas entre sí por al menos 3 veces su diámetro, con 1 a 3 puntos setígeros cerca de la inserción de la tibia, borde anterior con sedas largas; tibia media gradualmente dilatada hacia el ápice, con 9 a 11 espinas laterales pequeñas en la parte exterior, con 1 seda erecta y larga que sale de cada una; superficie dorsal chagrinada, con algunos hoyuelos suaves en la parte inferior cerca a la inserción de los tarsómeros y 1 quilla longitudinal con hoyuelos separados en toda su extensión, de cada uno saliendo 1 seda erecta y larga , la cual llega a la parte inferior externa; desde la parte apical interna hasta la parte inferior interna hay una hilera de hoyuelos profundos, de cada uno saliendo 1 seda; borde inferior con sedas largas muy unidas en toda su extensión; espolón interno llegando hasta la parte anterior del tercer tarsómero, espolón externo tan largo como el primer tarsómero, aplanados ventralmente y convexos dorsalmente, terminando en punta roma; con 5 tarsómeros los 4 primeros subtriangulares con una proyección en la parte apical del borde externo, aplanados dorsoventralmente, borde interno con sedas erectas largas y continuas, borde externo con 3 a 4 sedas y los ápices con mechones de sedas; primer tarsómero tan grande como el segundo al cuarto unidos, el quinto subcuadrangular tan largo como el tercero y cuarto unidos, terminando en 2 uñas simples, las cuales son rectas en la base y después curvadas, con 2 a 3 sedas en la mitad del tarsómero y densas en el borde apical. Sutura meso-metaesternal borrada en la mitad por un proceso del mesosterno, bordes laterales evidentes y brillantes, debajo de esta sutura hay una quilla lateral que forma una depresión en la parte anterior. Metaesterno con superficie brillante y chagrinada más fuerte en la parte anterior y a los lados inferiores debajo de la coxa; con puntos suaves sin sedas, separados al menos 3 veces su diámetro en la parte anterior y media, que hacia las zonas laterales se convierten en puntos ocelados, saliendo de cada uno 1 seda, separados 1 vez su diámetro. Parte media con una fóvea muy suave llegando hasta la parte posterior. Metaepisterno con superficie igual a la parte lateral del metasterno. Pata posterior con fémur, con sedas largas en el borde anterior, superficie lisa y brillante con puntos pequeños separados entre sí por al menos 3 veces su diámetro; tibia gradualmente dilatada hacia el ápice con 12-14 espinas pequeñas, cada una con 1 seda larga y erecta; superficie dorsal similar a la pata media; calcar tan largo como los tarsómeros primero y segundo unidos, aplanado ventralmente, con ápice truncado; con 5 tarsómeros con disposición similar a la pata media.

Abdomen. Esternitos abdominales glabros y chagrinados, desde el centro sale una hilera de puntos ocelados que se vuelve más densa hacia las zonas laterales; sexto esternito comprimido en la parte media; pigidio brillante con superficie chagrinada y puntos profundos separados entre sí por al menos 2 veces su diámetro, el reborde posterior está borrado en la parte central.

Órgano genital masculino. Edeago en vista lateral con phallobase subcuadrangular, parte basal con un abultamiento mediano a ambos lados, ápice con una constricción aproximada de $115^{\circ}$. Parámeros subtriangulares, más esclerotizados en la parte apical y con un proceso en forma de espina hacia la parte posterior (Fig. 1B). Dorsalmente simétricos con la base ensanchada y disminuyendo de grosor hacia la parte apical, borde externo curvado en la parte media, parte interna con un proceso que se ve desde más abajo de la parte apical de los parámeros dando la apariencia de un hueco, parte apical de los parámeros redondeada (Fig. 1C). En vista ventral, los parámeros presentan en la parte basal una curvatura que termina en una prolongación aguda; parte apical angosta, con ápices romos bien esclerotizados. Entre los parámeros e insertados por membrana en la parte media de los mismos, hay 2 procesos lameliformes esclerotizados sobrepuestos, el del parámero izquierdo encima del de la derecha (Fig. 1D). Saco interno tubular con ráspulas en el 1/3 basal; en el centro 1 lamela copulatriz grande subcuadrangular con 2 proyecciones en la parte lateral derecha bien esclerotizadas, superficie cubierta por cerdas en toda su extensión y en la parte izquierda presenta un proceso carnoso con cerdas largas (Fig. 1E). Parte apical con 3 lamelas accesorias, la central larga con forma no definida, más esclerotizada en el centro y con membrana semiquitinizada alrededor (Fig. 1F); la lateral izquierda en forma de $\mathrm{C}$ con la parte basal recta y bien esclerotizada, bordes laterales no definidos (Fig. 1G); la lateral derecha en forma de $\mathrm{L}$ ancha en la parte basal, bien esclerotizada en la parte derecha y zona apical, sin borde definido (Fig. 1H). Hembra. Longitud 12.8-13 mm; ancho 7.7-8 mm. Difiere del macho por el borde anterior de la cabeza prolongado (viéndose triangular) y redondeado, los dientes clipeales no son evidentes; en la frente, a nivel del borde anterior de los ojos, presenta un proceso cónico muy pequeño con 2 protuberancias pequeñas laterales (Fig. 1I). En el pro- 
noto no presenta la excavación como el macho, presenta un pequeño declive en la parte central cerca de la inserción de la cabeza (Fig. 1J). El sexto esclerito abdominal no está comprimido hacia la parte central.

Distribución. La especie se distribuye en Brasil en los estados de Espírito Santo y Minas Gerais, en los bosques cercanos al curso mediano y bajo del Río Doce (Fig. 3).

Comentarios. Con este estudio se amplía la distribución conocida de esta especie al estado de Minas Gerais. La especie está asociada a Mata atlántica perennifolia inferior a los $200 \mathrm{~m}$ de altitud en la cuenca del río Doce, ecosistema en estado crítico, por lo cual esta especie puede estar en peligro de desaparecer, como ya se mencionaba en la descripción original de la misma, donde Martínez argumentaba que esta especie estaba en peligro por la destrucción acelerada de su hábitat.

Dichotomius nemoricola (Pereira, 1942) (Fig. 2)

Holotipo. Museu de Zoologia, Universidade de São Paulo, São Paulo, Brasil (revisado).

Localidad tipo. Brasil, Rio Claro (Horto Florestal), Est. de São Paulo, 05.vi.1940, Leg. P. F. Pereira, excrem. bovinos. Material examinado (3수; 6 우우). Holotipo: Etiquetas: [1, etiqueta de color blanco con bordes negros] Rio Claro (S.P.) vi 1940 Coll. Claretiano [2, etiqueta rectangular roja con bordes negros] TIPO [3, etiqueta rectangular pequeña] + [4, etiqueta cuadrangular escrita a mano y en máquina] Pinotus nemoricola F. Pereira, 1941. P. Pereira det. 941 (1ㅇ). [MZSP]. Paratipo: idem (1 ㅇ) [MZSP]. Otro material: Brasil: Espírito Santo: Vargem Alta, ix.1995, J. Louzada. (2今; 2 ㅇ) [CEMT]; Vargem Alta, 680m, 15.ix.1995, J. Louzada (1 $\overbrace{}^{\Uparrow}$ [CEMT]; Minas Gerais: Cordisburgo, faz[enda] Pontinha, X-1995, F. Z. Vaz-de-Mello. (1 + ) [CEMT]; Diamantina. P[ar]q[ue] Est[adual] Rio Preto, 6-8-II-2001, S. L. Assis Jr. (1우) [CEMT].

Machos. Cuerpo de 14.5-16.8 mm de longitud y 9.2-9.5 $\mathrm{mm}$ de ancho (Fig. 2A). Coloración negra, con sedas castañas en los bordes laterales de la cabeza y pronoto.

Cabeza. Vez y media más ancha que larga, con sedas más largas en la parte posterior y de menor tamaño en la anterior. Margen anterior ovalado y truncado con un reborde en toda la extensión del clípeo, en el centro 2 dientes clipeales suaves. Superficie del clípeo en toda su extensión con arrugas suaves y convirtiéndose en puntos poco profundos cerca del proceso cefálico. Sutura clípeo-genal evidente, llegando hasta la base del proceso cefálico a la altura del borde anterior de los ojos. Genas con borde lateral recto y luego curvo, superficie similar a la del clípeo. Proceso cefálico ubicado en la parte anterior de la frente a nivel del borde anterior de los ojos, cónico, ancho en su base y terminando en un proceso de punta roma. Parte posterior de la frente con hoyuelos redondos y profundos separados entre sí 1 vez su anchura.
Tórax. Pronoto 2 veces más ancho que largo, con sedas evidentes en los bordes laterales. Superficie con puntuaciones pequeñas en la parte central y de mayor tamaño en los bordes laterales y posterior, separadas entre sí al menos 2 a 3 veces su diámetro. Parte anterior con un borde delgado y liso, que se comprime hacia los ángulos anteriores, los cuales son salientes y redondeados. Borde antero-lateral recto formando un ángulo de $120^{\circ}$ con el borde lateral posterior. En la parte central presenta una excavación que va desde la parte anterior hasta el $1 / 3$ del mismo, formando una protuberancia a cada lado. Fóveas laterales, alargadas y profundas, debajo de las mismas se forma un pliegue en la parte lateral. Hipómero con superficie chagrinada y puntos ocelados grandes separados una distancia de menos de 1 vez su diámetro, de algunos sale 1 seda erecta, larga y castaña; en la parte central debajo del fémur la superficie es brillante, parte lateral con hileras de sedas largas que sobresalen siendo evidentes en vista dorsal. Prosterno chagrinado con algunos puntos poco profundos en los bordes laterales. Coxas de las patas anteriores con superficie muy chagrinada y con hoyuelos en toda su extensión siendo más unidos en las partes laterales; fémur en vista ventral brillante, liso y con puntuaciones pequeñas separadas entre sí al menos 2 veces su diámetro, zona lateral cerca de la inserción de la tibia con 4 a 6 puntos setígeros fuertes, de cada uno sale 1 seda erecta y larga de color castaño; borde posterior con muchas sedas largas y castañas; desde la inserción del trocantín salen 2 quillas paralelas al fémur, una llega a la parte apical y la otra al surco del fémur con sedas en toda su extensión que se vuelven más densas hacia el surco; tibia con 4 dientes laterales y superficie chagrinada, parte media más elevada, de allí salen 3 quillas hacia los dientes tibiales anteriores; en la parte central hay una hilera de puntos setígeros grandes y profundos; de cada uno sale 1 seda erecta y larga, luego continua una quilla en el borde externo también con sedas, interrumpida en los dientes tibiales; espolón tibial en forma de L invertida, va hasta el tercer tarsómero, aplanado ventralmente y convexo en la parte dorsal; con 5 tarsómeros, el primero y quinto subcuadrangulares, tan largos como el segundo y tercero unidos, los cuales son subovoides, con sedas en el borde apical; el quinto en el borde apical y ventral con sedas largas y termina en 2 uñas simples. Élitros con la superficie de las interestrías chagrinada con puntos poco profundos y pequeños, separados entre sí por al menos 3 veces su diámetro; estrías bicarenadas con puntos ocelados pequeños en toda su extensión, separados entre sí por aproximadamente 3 veces su diámetro; húmero pronunciado en la parte anterior de las estrías 6 y 7 . Epipleura separada por una carena fuerte, de superficie chagrinada. Mesosterno reducido en su parte media, en la cual hay un proceso liso y brillante que no deja ver en su parte central la sutura 

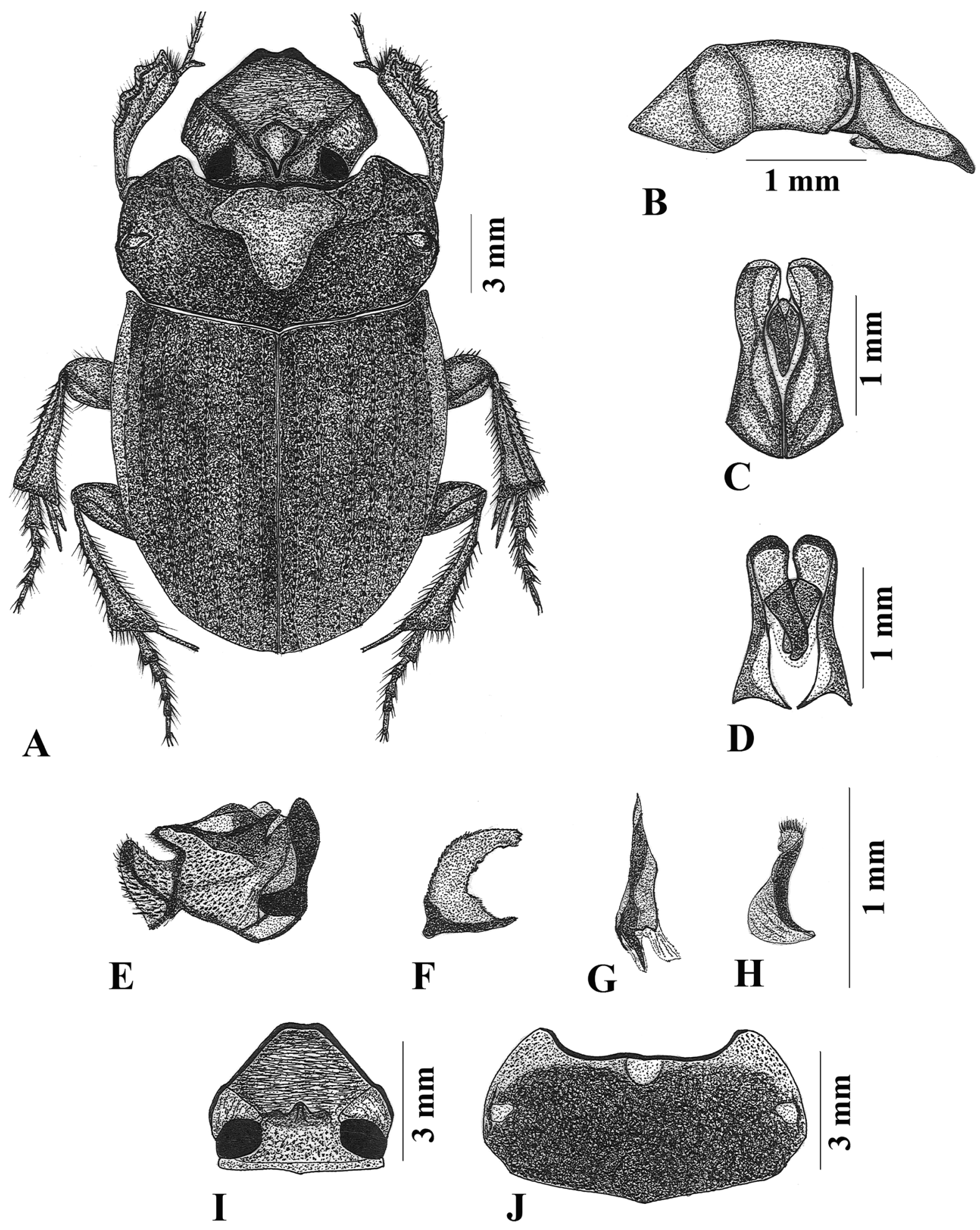

Figura 1. Dichotomius camposeabrai. A, Habitus macho; B, edeago vista lateral; $\mathrm{C}$, parámeros vista dorsal; D, parámeros vista ventral; E, lamela copulatriz; F, primera lamela accesoria; G, segunda lamela accesoria; H, tercera lamela accesoria; I, cabeza hembra; J, pronoto hembra. 


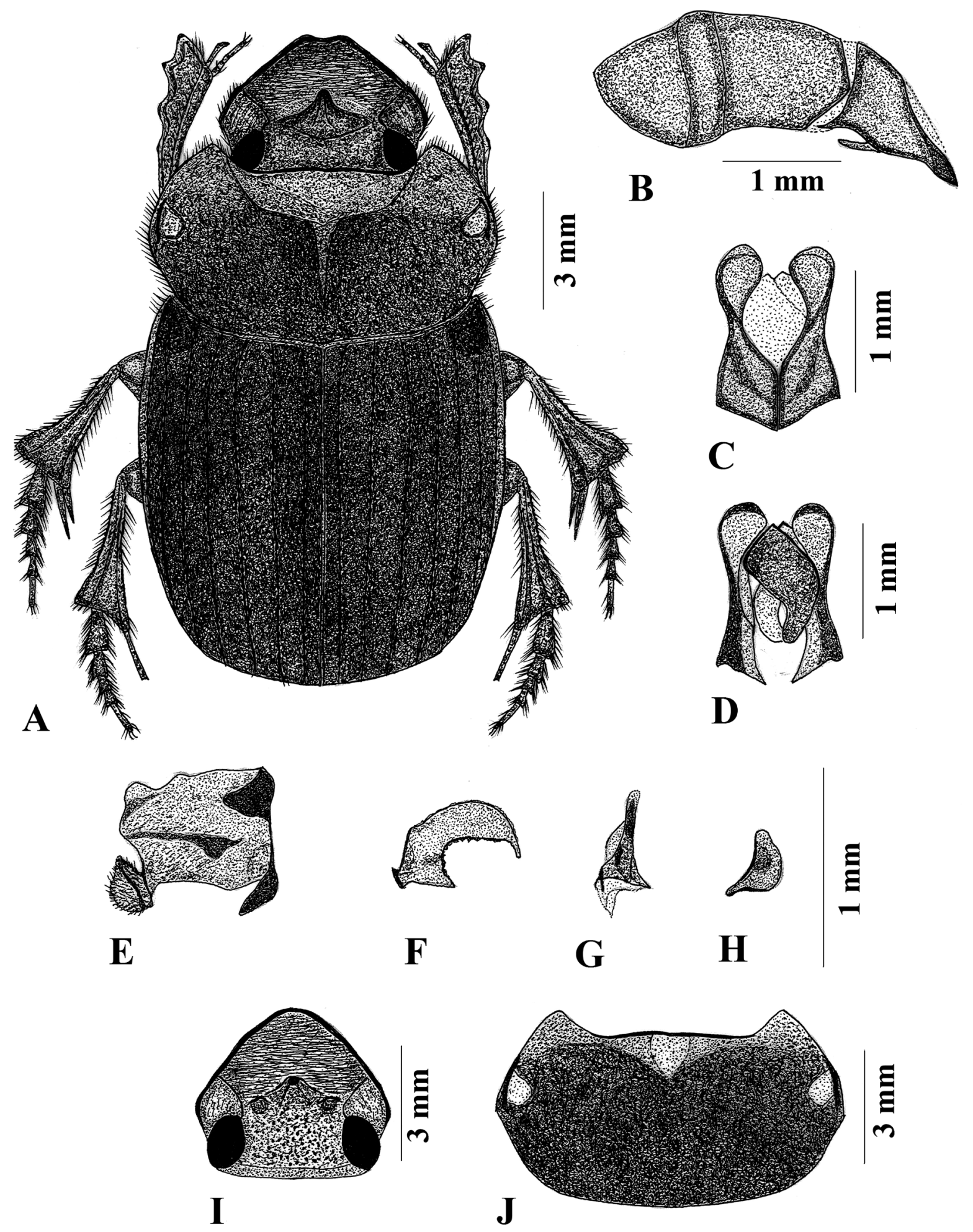

Figura 2. Dichotomius nemoricola. A, Habitus macho; B, edeago vista lateral; C, parámeros vista dorsal; D, parámeros vista ventral; E, lamela copulatriz; F, primera lamela accesoria; $\mathrm{G}$, segunda lamela accesoria; H, tercera lamela accesoria; I, cabeza hembra; J, pronoto hembra. 


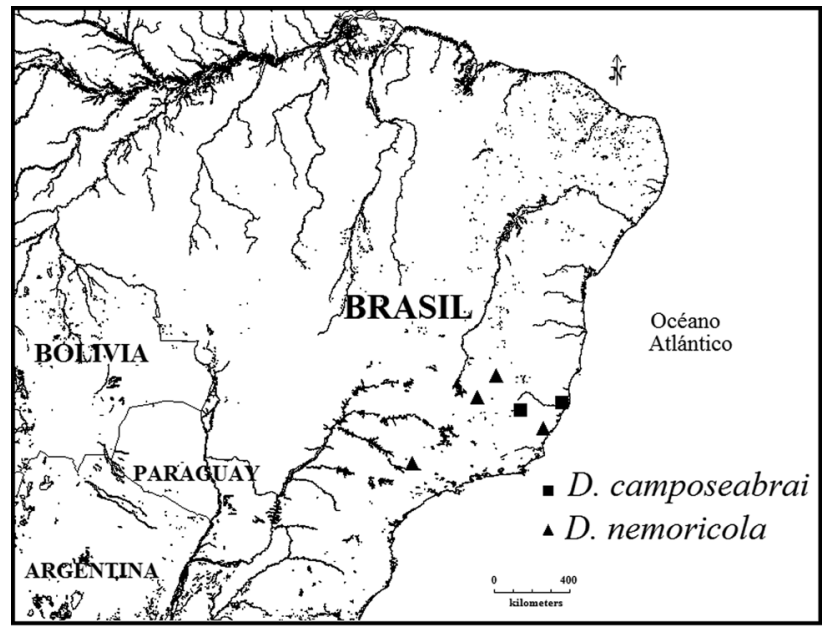

Figura 3. Mapa de distribución de Dichotomius camposeabrai y D. nemoricola

meso-metaesternal, hacia los lados presenta una superficie chagrinada con puntos ocelados grandes, separados menos de su diámetro, de algunos saliendo sedas. Mesepisterno con la misma superficie que los bordes laterales del mesosterno, pero de cada punto ocelado sale 1 seda erecta y larga. Patas medias con coxas con superficie chagrinada, con puntos suaves en la parte media y ocelados en la parte lateral superior. Fémur medio en vista ventral con superficie brillante, lisa y con puntuaciones pequeñas separadas entre sí por al menos 3 veces su diámetro; borde anterior con algunas sedas cortas en la parte apical. Tibia media gradualmente dilatada hacia el ápice, con 9 a 11 espinas laterales pequeñas en la parte exterior, de cada una sale 1 seda erecta y larga; superficie dorsal chagrinada, con 1 quilla longitudinal con hoyuelos separados en toda su extensión, de cada uno saliendo 1 seda erecta y larga, la cual llega a la parte inferior externa. Desde la parte apical interna hasta la inferior interna hay una hilera de hoyuelos profundos, de cada uno saliendo 1 seda. Borde inferior con sedas largas muy unidas en toda su extensión. Espolón interno llegando hasta la parte anterior del tercer tarsómero, espolón externo tan largo como el primer tarsómero, ambos aplanados ventralmente y convexos dorsalmente, terminando en punta roma; con 5 tarsómeros, los 4 primeros subtriangulares con una proyección en la parte apical del borde externo, aplanados dorsoventralmente, borde interno con sedas erectas largas y continuas, borde externo con 3 a 4 sedas y los ápices con mechones de sedas en el ápice. Primer tarsómero tan grande como el segundo y tercero unidos, el quinto subcuadrangular tan largo como el tercero y cuarto unidos, terminando en 2 uñas simples rectas en la base y después curvadas, con 2 a 3 sedas en la mitad del tarsómero y densas en el borde apical. Sutura meso-metaesternal borrada en la mitad por un proceso del mesosterno, bordes laterales evidentes y brillantes, debajo de esta sutura hay una quilla lateral que forma una depresión en la parte anterior. Metaesterno con superficie brillante y chagrinada más fuerte en la parte anterior y a los lados inferiores debajo de la coxa; con puntos poco profundos sin sedas separados entre sí por al menos 3 veces su diámetro en la parte anterior y media, que se convierten hacia las zonas laterales en puntos ocelados, saliendo de cada uno 1 seda, separados entre sí 1 vez su diámetro. Parte media con una fóvea muy suave que llega hasta la parte posterior. Metaepisterno con superficie igual a la parte lateral del metasterno. Pata posterior con fémur con sedas largas en el borde anterior, con superficie lisa y brillante con puntos pequeños separados al menos 3 veces su diámetro. Tibia posterior gradualmente dilatada hacia el ápice con 12-14 espinas pequeñas, cada una con 1 seda larga y erecta. Superficie dorsal similar a las patas media; calcar tan largo como el primer y segundo tarsómeros unidos, aplanado ventralmente, con ápice truncado, con 5 tarsómeros con disposición similar a la pata media. Abdomen. Esternitos abdominales glabros y chagrinados, desde el centro sale una hilera de puntos ocelados que se vuelven más densos hacia las zonas laterales. Sexto esternito comprimido en la parte media. Pigidio brillante con superficie chagrinada y puntos profundos separados entre sí 2 veces su diámetro, el reborde posterior está borrado en la parte central.

Órgano genital masculino. Edeago en vista lateral con phallobase subcuadrangular, parte basal con un abultamiento mediano a ambos lados, ápice con una constricción aproximada de $115^{\circ}$. Parámeros subtriangulares, más esclerotizados en la parte apical y con un proceso en forma de espina hacia la parte posterior (Fig. 2B). Dorsalmente simétricos con la base ensanchada y disminuyendo de grosor hacia la parte apical, borde externo curvado en la parte media, parte interna con un proceso que se ve desde más abajo de la parte apical de los parámeros, ápice de los parámeros redondeado (Fig. 2C). En vista ventral los parámeros en la parte basal presentan una curvatura y después termina en una prolongación aguda, parte apical angosta con ápices romos y redondeados bien esclerotizados. Entre los parámeros, e insertados abajo de la parte apical hasta 3/4 de la parte basal por membrana, hay 2 procesos lameliformes esclerotizados sobrepuestos, el del parámero izquierdo encima del de la derecha (Fig. 2D). Saco interno tubular con ráspulas en el 1/3 basal; en el centro lamela copulatriz grande subcuadrangular con 2 proyecciones en la parte lateral derecha bien esclerotizadas, superficie cubierta por cerdas en toda su extensión y en la parte izquierda presenta un proceso carnoso con cerdas largas (Fig. 2E). Parte apical con 3 lamelas accesorias, la central de forma alargada, más esclerotizada en el centro y con membrana semiquitinizada alrededor (Fig. 2F). La lateral izquierda en forma 
de $\mathrm{C}$ con la parte basal recta y bien esclerotizada, con una proyección en la parte izquierda, bordes laterales no definidos (Fig. 2G). La lateral derecha sin forma definida, bien esclerotizada y con bordes definidos (Fig. 2H).

Hembra. 13.8-16.8 $\mathrm{mm}$ de longitud y $8.6-9.4 \mathrm{~mm}$ de ancho. Difiere del macho por presentar el borde anterior de la cabeza prolongado (viéndose triangular) y redondeado, dientes clipeales no evidentes; en la frente, a nivel del borde anterior de los ojos presenta un proceso cónico muy pequeño con 2 protuberancias laterales pequeñas (Fig. 2I). En el pronoto no presenta la excavación como el macho, presenta un pequeño declive en la parte central cerca de la inserción de la cabeza (Fig. 2J). El sexto esclerito abdominal no está comprimido hacia la parte central.

Distribución. Se distribuye en Brasil en los estados de Espírito Santo, Minas Gerais y São Paulo (Fig. 3).

Comentarios. Los ejemplares de esta especie fueron recolectados en distintos hábitats de distintos biomas (Cerrado y Mata atlántica), y en cada uno de ellos en apenas una ocasión (aunque se hicieron numerosas recolecciones en cada uno de estos sitios, en distintas épocas y con distintas metodologías). Esto puede indicar algún tipo de hábitat muy especializado, por ejemplo asociación con letrina o nido de algún mamífero.

\section{Discusión}

Martínez (1974) incluyó a $D$. camposeabrai dentro del grupo buqueti, ubicándola como la especie hermana de $D$. quadrinodosus (Felsche, 1901), dada las características de la armadura cefálica, armadura pronotal y élitros. Sin embargo, recientemente Arias-Buriticá (2011) realizó un estudio, en el que se incluyen las características del órgano genital masculino, encontrando que la relación entre estas 2 especies no se da. Dichotomius camposeabrai presenta 2 procesos lameliformes entre los parámeros (Fig. 1B), los cuales no presenta $D$. quadrinodosus, especie que es parte del grupo reclinatus, en el cual una de las principales características es la no presencia de estos procesos entre los parámeros (Arias-Buriticá y Vaz-de-Mello, in litt).

Al comparar D. camposeabrai con otras especies del género, se propone la transferencia de ésta al grupo bitiensis, dadas las similitudes que presenta con $D$. nemoricola (especie de la que era desconocido el macho descrito en este estudio) en caracteres de los órganos genitales masculinos, como la forma de los parámeros y presencia de los procesos lameliformes (Figs. 1D-F, 2D-F), lamela copulatriz típica, la cual tiene un proceso carnoso con cerdas largas en la parte izquierda (Figs. 1G, 2G) y las lamelas accesorias (Figs. 1H-J, 2H-J); así como por características de la morfología externa, como la forma lateral del pronoto, la punturación pronotal, estrías e interestrías elitrales y la punturación y superficie del hipómero, el pro, meso y metaepisterno; por lo cual, la relación de estas especies sería más evidente que la propuesta por Martínez (1974) entre D. camposeabrai y D. quadrinodosus. Otro carácter que refuerza la inclusión de $D$. camposeabrai dentro del grupo bitiensis es la morfología de la hembra, descrita por primera vez en este estudio, la cual presenta el margen de la cabeza prolongado en forma casi triangular con dientes clipeales poco evidentes y el pronoto con un declive en la parte anterior del pronoto, que son 2 de las principales características que definen las especies de la entonces "sección bitiensis" (Pereira, 1942).

El grupo bitiensis fue revisado por Pereira (1942). Se recomienda realizar una nueva revisión para confirmar los caracteres aquí expuestos en las otras especies pertenecientes a este grupo (por ejemplo, D. bitiensis, D. motai y D. rugosicollis) y redefinir la sección teniendo en cuenta tanto la morfología externa como la de los órganos genitales masculinos.

\section{Agradecimientos}

A François Génier (CMNC), por el envío del material de D. camposeabrai, y a Sonia Casari y Carlos Campaner (MZSP), por su colaboración durante nuestra visita. A Jesús Orlando Rangel-Ch. y al grupo de Investigación Biodiversidad y Conservación (Universidad Nacional de Colombia), por la colaboración y apoyo brindado. A Mateus Souza, Ricardo Vicente y Miquéias Ferrão (UFMT), por toda su colaboración y hospitalidad con el primer autor. A Jhon Neita por su asesoramiento en las ilustraciones. A los evaluadores anónimos que enriquecieron este escrito. FZVM es becario de productividad de CNPq (PQ 304925/2010-1) y agradece además el apoyo de FAPEMAT (570847/2008 y 447441/2009).

\section{Literatura citada}

Arias-Buriticá, J. 2011. Revisión taxonómica de la sección "buqueti", Dichotomius Hope, 1838 (Coleoptera: Scarabaeidae: Scarabaeinae). Tesis Maestría. Facultad de Ciencias, Universidad Nacional de Colombia, Bogotá. 129 p. Edmonds, W. 1972. Comparative skeletal morphology, systematics and evolution of the Phanaeine dung beetles (Coleoptera: Scarabaeidae). The University of Kansas Sciencie Bulletin 45:731-874.

Felsche, C. 1901. Beschreibungen coprophager Scarabaeiden. Deutsche Entomologische Zeitschrift. p. 135-155.

Gandini, P. y C. Aguilar. 2009. Seis nuevas especies de Dichotomius Hope, 1838 de Sudamérica y descripción del macho de Dichotomius camargoi Martínez, 1956. Giornale Italiano di Entomologia 12:135-164. 
Génier, F. 2000. Dichotomius comarapensis sp. nov., une nouvelle espèce bolivienne de scarabée brachyptère (Coleoptera: Scarabaeidae: Scarabaeinae). Fabreries 25:25-31.

Hijmans, R., L. Guarino, C. Bussink, P. Mathur, M. Cruz, I. Barrantes y E. Rojas. 2004. DIVA GIS ver. 5.2, Sistema de información geográfica para el análisis de datos de distribución de especies. www.div a-gis.org; última consulta: 2.VII.2011.

Kohlmann, B. 2003. Tribu Coprini. In Atlas de los escarabajos de México, Coleoptera Lamellicornia, vol. 22, M. A. Morón (ed.). Argania, Barcelona. p. 45-57.

Luederwaldt, H. 1929. As espécies brasileiras do gênero Pinotus (Coleoptera: Lamellicornidae: Coprini) com algumas considerações também sôbre outras espécies. Revista do Museo Paulista 16:603-775.

Martínez, A. 1974. Una nueva especie de Dichotomius s. str.
(Coleoptera, Scarabaeidae, Coprini, Ateuchina). Physis sección C 33:41-45.

Medina, C., C. Scholtz y B. Gill. 2003. Morphological variation and systematics of Canthon and related genera of new world Canthonini dung beetles. Deutsche Entomologische Zeitschrift 50:23-68.

Pereira, F. 1942. Pinotus da secção Bitiensis. Papéis Avulsos do departamento de Zoologia, São Paulo-Brasil 2:117-131.

Vaz de Mello, F., J. Louzada y M. Gavino. 2001. Nova espécie de Dichotomius Hope, 1838 (Coleoptera, Scarabaeidae) do Espírito Santo, Brasil. Revista Brasileira de Entomologia 45:99-102.

Zunino, M. 1978. L'armatura genital negli Onthophagini Techiche de preparazione e criteri di studio (Coleoptera, Scarabaeoidea). Bolletino della Società Entomologica Italiana, Supplemento 90:21-26. 